Rabaska

Revue d'ethnologie de l'Amérique française

\title{
Chaire de recherche du Canada en oralité des francophonies minoritaires
}

\section{Jean-Pierre Pichette}

Volume 8, 2010

URI : https://id.erudit.org/iderudit/045297ar

DOI : https://doi.org/10.7202/045297ar

Aller au sommaire du numéro

Éditeur(s)

Société québécoise d'ethnologie

ISSN

1703-7433 (imprimé)

1916-7350 (numérique)

Découvrir la revue

Citer ce document

Pichette, J.-P. (2010). Chaire de recherche du Canada en oralité des

francophonies minoritaires. Rabaska, 8, 285-290.

https://doi.org/10.7202/045297ar d'utilisation que vous pouvez consulter en ligne.

https://apropos.erudit.org/fr/usagers/politique-dutilisation/ 


\section{Rapports des institutions}

\section{ACADIE}

\section{Chaire de recherche du Canada en oralité des francophonies minoritaires}

COFRAM / Centre acadien

Téléphone : (902) 769-2114

Université Sainte-Anne

Télécopieur : (902) 769-0063

Pointe-de-l'Église_Courriel : jeanpierre.pichette@usainteanne.ca

(Nouvelle-Écosse) B0W 1M0 Toile : www.usainteanne.ca/cofram

\section{Présentation}

Le sixième bilan annuel des activités de la Chaire de recherche en oralité des francophonies minoritaires d'Amérique (COFRAM) reste, on le notera aisément, en symbiose avec les objectifs inscrits dans la mission de la chaire autant qu'en continuité avec les chantiers qu'elle a lancés. Le titulaire a concentré son attention sur le travail d'animation du Laboratoire de littérature orale (LABOR), principalement autour du projet ÉCLORE, objet d'une nouvelle demande de subvention au Conseil de recherches en sciences humaines (CRSH) - d'ailleurs fructueuse -, et sur son programme de diffusion, notamment par l'édition des ouvrages publiés en cours d'année ou à paraître : publication des actes des journées internationales d'étude sur l'Édition des contes de tradition orale de l'automne 2008, coordination de la revue d'ethnologie Rabaska, collaboration à la relance des Cahiers Charlevoix chez un nouvel éditeur et à la préparation du manuscrit final du Dictionnaire des écrits de l'Ontario français (DÉOF). Pour accomplir l'essentiel de la programmation régulière, le titulaire est assisté par une équipe locale importante, le personnel du Centre acadien où loge sa chaire, les auxiliaires de recherche du LABOR et les chercheurs du Groupe de recherche en études acadiennes (GRÉA); les collaborateurs de l'extérieur sont encore plus nombreux, auteurs des divers écrits publiés dans les ouvrages collectifs, collègues ethnologues de la Société québécoise d'ethnologie ( $\mathrm{S} \mathrm{QE})$, confrères franco-ontariens de la Société Charlevoix, partenaires du Centre de recherche bretonne et celtique (CRBC) et autres experts internationaux dont on lira les noms dans ces travaux. Tous ont droit à notre gratitude. 


\section{Réunions scientifiques}

A. ATELIERS BRITTO-FRANCO-CANADIENS - Le passage à Québec d'une délégation de la Ville de Brest, en mission officielle, a permis d'organiser, les 12 et 13 avril 2010, un troisième atelier britto-franco-canadien avec les membres présents du Centre de recherche bretonne et celtique (CRBC), de l'Université de Bretagne occidentale, et la participation de la Société québécoise d'ethnologie ( $\mathrm{SeE}$ ) et de la Cofram. Cette réunion s'inscrivait dans les échanges établis avec ces partenaires, notamment dans le chantier « Du folklore à l'ethnologie. Parcours comparés de la Bretagne et du Canada francophone » qui a accompagné, sur une base régulière depuis l'année 2000, de nombreuses manifestations (colloques, journées d'étude, publications). Cette fois, dans la continuité des ateliers exploratoires de décembre 2007 et d'avril 2009, nous avons discuté de la planification d'un colloque international conjoint sur le thème des prêtres ethnographes et des modalités d'organisation d'une telle rencontre qui se tiendrait en deux lieux, à Brest et à Pointe-del'Église, au cours de 2011. Une autre réunion, prévue à la fin de l'été, devrait permettre de parachever la mise en place de cet événement, dont le thème s'avère très engageant.

B. Chercheurs invités - Les quatre chercheurs que la Cofram a accueillis cette année partageaient le même intérêt pour la chanson traditionnelle. Le passage à la baie Sainte-Marie, les 12 et 13 mai 2009, de M. Ronald Labelle, titulaire de la chaire de recherche McCain en ethnologie acadienne à l'Université de Moncton, a été l'occasion d'une séance de travail sur nos projets communs ; son séjour coïncidait avec le lancement à Pubnico de son édition des chansons acadiennes qu'Helen Creighton avaient recueillies en Nouvelle-Écosse : Chansons de Pubnico et Grand-Étang (2008), un cahier dont la Cofram a été partenaire. Dans le cadre d'une tournée qu'elle effectuait au Québec et en Acadie, $\mathrm{M}^{\mathrm{me}}$ Marlène Belly s'est arrêtée à l'Université SainteAnne du 2 au 5 novembre 2009. Ethnomusicologue, chargée d'enseignement à l'Université de Poitiers, elle centre ses travaux sur la chanson française de tradition orale (notamment le répertoire franco-canadien) dont elle examine les processus de variation et le principe de composition sur timbre. En lien avec la Bibliothèque nationale de France, elle participe à l'édition des fichiers Coirault. Le 4 novembre, $\mathrm{M}^{\mathrm{me}}$ Belly livra aux membres du Groupe de recherche en études acadiennes la quatrième conférence de la COFRAM, qu'elle avait intitulée "Chansons de tradition orale francophones. L'art du "médaillon"». Cet exposé et les séances de discussion qui l'ont encadré déboucheront prochainement sur des projets de collaboration. Au semestre d'hiver, deux autres visiteurs se sont en outre adressés aux étudiants du cours Ethn/Fran 2023 Littérature orale II : la chanson folklorique et les formes 
brèves. Les 4 et 5 février 2010, M. André Gervais, professeur de littérature à l'Université du Québec à Rimouski, auteur et éditeur, présentait le premier livre personnel de Luc Lacourcière, Essais sur Émile Nelligan et sur la chanson populaire (Fides, 2009), une publication posthume, préparée par M. Gervais, qui met en relief les deux fronts où Lacourcière est un pionnier : en culture populaire, il est le pionnier au Canada de l'enseignement universitaire du folklore, et, en culture savante, il est le pionnier au Québec de l'édition critique avec son édition des poésies complètes d'Émile Nelligan. Il donna aussi la cinquième conférence de la COFRAM à la galerie du Rendezvous de la Baie : "De la chanson et de la poésie : ressemblances et différences ». À son tour, M. Marcel Bénéteau, professeur d'ethnologie à l'Université de Sudbury et associé à divers titres aux activités de la COFRAM, était notre hôte les 11 et 12 mars 2010. Spécialiste du patrimoine oral du Sud-Ouest, la plus ancienne région de l'Ontario, il fit part aux étudiants des recherches entreprises pour reconstituer le répertoire de la chanson traditionnelle et mit en vedette plusieurs échantillons de ce vaste fonds musical. Il prononça la sixième conférence de la COFRAM, qui prit la forme d'une conférence-récital : " "On commence à s'en souviendre" : à la recherche du répertoire traditionnel de la chanson de la rivière Détroit ».

\section{Laboratoire de littérature orale}

A. DÉPôTs - On a noté un accroissement sensible des archives orales durant l'exercice 2009-2010. L'augmentation de la fréquentation de nos cours d'ethnologie y est certainement pour une bonne part puisque ce sont les collections préparées par nos étudiants dans le cadre de leur apprentissage qui composent l'essentiel de ces acquisitions nouvelles. Les 39 collections étudiantes, déposées en 72 versements, forment un total de 1327 pièces de littérature orale principalement, chansons, formes brèves et récits traditionnels, distribuées en 1197 enregistrements et 130 manuscrits. Les captations vidéo de la cinquième édition du Festival de la parole de la baie Sainte-Marie (2009) et des témoignages réunis pour cette occasion par André Gaudet sur un humoriste acadien (Souvenirs d'Adélard à Jos Doucet, Société acadienne de Clare et Festival de la parole de la Baie Sainte-Marie, 2009), réalisées toutes deux par notre technicien, complètent la série de documents archivés cette année au Centre acadien.

B. La baSe de tRaitement ÉClore - À la collection sonore de Jean-Pierre Pichette, déjà insérée en entier dans un compartiment de la base ÉClORE, s'est ajoutée sa collection manuscrite, un corpus de 3695 textes : tous les documents narratifs (contes et histoires brèves, légendes, mystifications) et les descriptions de jeux traditionnels ont été saisis dans un deuxième 
compartiment de la base de données et versés dans le système; on vient d'entreprendre le traitement des dernières liasses de manuscrits non numérotés portant sur des genres brefs de littérature orale. Ce classement de tous les ethnotextes de cette collection a depuis servi à constituer une série d'outils de travail : inventaire de la collection sonore, répertoires narratifs régionaux par genres, par lieux de collecte, par informateurs ; tous ces dossiers faciliteront la sélection des textes et la planification des tâches aux étapes ultérieures. Avec la publication du protocole de transcription ( $c f$. infra les actes des journées d'étude de 2008), les travaux préliminaires du projet ÉcLo$\mathrm{RE}-$ « De l'oral à l'écrit. Établissement et édition d'un corpus de littérature orale »- sont maintenant achevés. Aussi, la subvention ordinaire du CRSH, obtenue ce printemps pour les trois prochaines années, a-t-elle permis de lancer le travail d'édition. La publication des premiers répertoires de cette collection pourra bientôt commencer.

\section{Publications}

A. RABASKA - Le 11 décembre 2009, au moment de son banquet annuel à l'hôtel Gouverneur de Québec, la Société québécoise d'ethnologie fit le lancement officiel du septième volume de Rabaska, revue d'ethnologie de l'Amérique française (Québec, SQE, vol. 7, 2009, 333 p.). Une soixantaine d'auteurs ont encore une fois collaboré à cette publication annuelle et produit études (5 articles et terrains), portrait de chercheur, points de vue croisés (4), résumés de colloques (2), nécrologies (3), comptes rendus de livres (29) et d'expositions (2), rapports d'institutions (15), chroniques sur les honneurs (6) et les mémoires et thèses (25). En plus de la coordination de la revue, de la saisie et de l'uniformisation des textes à la COFRAM, la participation de la petite équipe du LABOR à Rabaska est toujours significative. Nos assistants y œuvrent à peu près tous à des titres divers, notamment en rédigeant quelques rubriques, et des collègues de l'Université Sainte-Anne, membres du GrÉA, y coopèrent aussi parfois par un article, surtout par des comptes rendus. Grâce à une entente avec la plateforme Érudit (www.erudit.org), les abonnés ont accès à notre revue en version électronique, en plus de la version imprimée, pour les volumes récents $(5,6$ et 7$)$; de plus, la numérisation rétrospective des volumes 1 à 4 étant achevée depuis le printemps 2010, l'intégralité de la revue Rabaska est désormais accessible en ligne.

B. CAHIERs CharlevolX - Les neuf chercheurs de la Société Charlevoix, un groupe qui se consacre à l'étude de l'Ontario français, a conclu, en mars 2009, une entente avec les Presses de l'Université d'Ottawa (Puo) et le Centre de recherche en civilisation canadienne-française ( $\mathrm{CRCCF}$ ) pour l'édition de 
ses Cahiers Charlevoix. La publication de cette série a donc repris son rythme en mars 2010 avec la livraison de sa plus récente cuvée : les Cahiers Charlevoix 8. Études franco-ontariennes (Ottawa, Société Charlevoix et Puo, 2010, 252 p.). J.-P. Pichette, qui est l'un des cofondateurs de cette société en signe l'avant-propos (p. 5-8) et présente la troisième et ultime partie de son étude sur la danse de l'aîné célibataire : "Le Principe du limaçon ou la résistance des marges. Essai d'interprétation de la dynamique des traditions » (p. 11-75). Il conclut sa réflexion par la proposition d'un modèle tout simple capable d'apporter un éclairage original et significatif sur les rapports entre centre et périphérie. Simon Laflamme, de l'Université Laurentienne, Michel Bock et France Martineau, de l'Université d'Ottawa, y donnent aussi des études substantielles, tandis que Michel Gaulin, le secrétaire de la Société Charlevoix, y tient la chronique.

C. Actes des journées internationales D'Étude. Les comptes rendus de l'atelier consacré à l'édition des récits de tradition orale, qui s'est déroulé à Pointe-de-l'Église à l'automne 2008, viennent de paraître. Comme les actes des colloques précédemment organisés par la COFRAM, ceux-ci rassemblent la totalité des points de vue exposés au cours de cette rencontre internationale et sont publiés dans la revue Port Acadie: Éditer des contes de tradition orale. Pour qui ? Comment? Actes des journées internationales d'étude tenues les 23 et 24 octobre 2008 à l'Université Sainte-Anne (Port Acadie Revue interdisciplinaire en études acadiennes, $\mathrm{n}^{\text {os }} 16-17$, automne 2009printemps 2010, 273 p.). Disposées selon les séances de l'atelier, les quatorze interventions examinent les destinataires visés (Transcrire pour qui ?), les problèmes engendrés par le passage des contes oraux à l'écrit (Transcrire, traduire ou récrire?) et le traitement des documents manuscrits et des témoignages littéraires incomplets, résumés ou retouchés (Comment éditer en l'absence de l'oral ?) ; enfin, la synthèse des positions avancées forme la section finale (Comment transcrire et éditer? Convergences) dans laquelle J.-P. Pichette dresse le bilan des méthodes employées depuis un siècle (« De l'oral à l'écrit : toute une histoire ») et renouvelle la formulation d'un «Protocole pour la transcription des documents de source orale en vue de l'édition ». Cette publication, fruit d'un partenariat avec la Chaire de recherche McCain en ethnologie acadienne (CREmeA, Université de Moncton), le département de Folklore et ethnologie de l'Amérique française (Dfeus, Université de Sudbury), le Centre interuniversitaire de recherche en littérature et en culture québécoises (CRILCQ, Université Laval) et le Centre de recherche bretonne et celtique (CRBC, Université de Bretagne occidentale), clôt la phase préparatoire au projet d'édition d'un corpus de littérature orale (ÉCLORE). 
D. DictionNAIRE DES ÉCRITS DE L'ONTARIO FRANÇAIS - L'année 2009 aura été marquée par la relance et l'achèvement de cet ambitieux projet, modestement lancé en 1982 par une équipe de chercheurs de Sudbury. Le Dictionnaire des écrits de l'Ontario français, codirigé par Gaétan Gervais de l'Université Laurentienne et J.-P. Pichette, recense, par des articles descriptifs, tous les ouvrages autonomes parus en français, depuis le Quatriesme voyage du Sieur de Champlain [...] en la Nouvelle France, fait en l'année 1613, jusqu'aux écrits beaucoup plus nombreux de l'année 1993, soit une amplitude de trois cent quatre-vingt-un ans. Pour cette période, on a dûment rassemblé toutes les publications de langue française dont l'auteur est né en Ontario, ou y a vécu et publié, ou qui ont l'Ontario comme sujet. Le Dictionnaire des écrits de l'Ontario français (1613-1993) compte précisément 2537 œuvres, rédigées durant ces quatre siècles par un millier d'auteurs et présentées dans l'ordre alphabétique de leurs titres. Pour y parvenir, l'équipe de direction a, pendant le quart de siècle qu'a duré l'entreprise, recruté une dizaine d'équipes d'assistants et de nombreux rédacteurs. Environ deux cents collaborateurs ont ainsi œuvré à ce collectif unique dont la publication est prévue pour l'automne 2010, en parfait synchronisme avec les commémorations du $400^{\mathrm{e}}$ anniversaire de la présence française en Ontario.

\section{Autres}

Le portail www.usainteanne.ca/cofram contient le sommaire des activités de la chaire, notamment les bulletins d'information préparés au Centre acadien : La Mouvée, bulletin d'information de la CofRAM ( ${ }^{\circ}$ 5, décembre 2009-janvier 2010, 8 p.) et le GRÉA (n ${ }^{\circ}$ 6, hiver 2010, 12 p.).

JeAn-Pierre Pichette

\section{Chaire de recherche McCain en ethnologie acadienne}

CREMEA, Département d'études françaises

Université de Moncton

Téléphone : (506) 858-4412

Moncton (Nouveau-Brunswick)

E1A 3E9

Courriel : ronald.labelle@umoncton.ca

Toile : www.umoncton.ca/crmea

\section{Présentation}

La CREMEA continue à remplir son mandat de mettre en valeur les ressources patrimoniales acadiennes par la réalisation d'ouvrages destinés à un public varié, ainsi que de promouvoir la place de l'ethnologie au sein du Département 